\title{
Quantitative Three-Dimensional Analysis of the Catecholaminergic Innervation of Identified Neurons in the Macaque Prefrontal Cortex
}

\author{
Leonid S. Krimer, Robert L. Jakab, and Patricia S. Goldman-Rakic \\ Section of Neurobiology, Yale University School of Medicine, New Haven, Connecticut 06510
}

The present study provides a complete quantitative threedimensional analysis of neurons in primate prefrontal cortex targeted by catecholaminergic axons. Individual pyramidal and nonpyramidal cells in fixed slices were filled with Lucifer yellow (LY) and recovered with anti-LY antibody combined with antityrosine hydroxylase $(\mathrm{TH})$ antisera to reveal catecholaminergic axons. The total number of $\mathrm{TH}$ contacts and $\mathrm{TH}$ apposition density (THAD) was obtained for pyramidal and nonpyramidal cells in different layers. Four TH contacts (two on spines and two on shafts) were selected for correlated electron microscopic examination and serially sectioned; all four were confirmed as membrane appositions. Quantitative analysis revealed $90 \mathrm{TH}$ contacts per pyramidal neuron in layer III, with a density of 0.8 per $100 \mu \mathrm{m}$ of dendritic length (i.e., averaging one contact per basal dendrite). Remarkably, pyramids of layers III, $\mathrm{V}$, and $\mathrm{VI}$ had the same THAD values, with a highly regular distribution of $\mathrm{TH}$ terminals on their spiny dendritic trees. In contrast, TH contacts on nonpyramidal neurons in layer III were half as dense and, moreover, were distributed irregularly and showed large variation from cell to cell. Neurons in layers II and superficial III had the highest THAD, as compared with deeper layers (1.4 vs 0.7 per $100 \mu \mathrm{m}$ of dendritic length for pyramids; 0.53 vs 0.4 for interneurons). The highly organized TH innervation of pyramidal neurons, with at least one contact on virtually every dendrite, indicates that catecholaminergic, presumably dopaminergic, terminals are placed strategically along the entire dendritic tree to modulate most, if not all, of the excitatory input of a neuron. At the same time, the sparsity of contacts per dendrite may explain cortical vulnerability in diseases involving dopamine.

Key words: dopamine; tyrosine hydroxylase; medial prefrontal cortex; projection neurons; GABAergic neurons; Lucifer yellow; immunohistochemistry; fixed slice video microscopy
The importance of dopamine neurotransmission in the highest cognitive functions, particularly those of the prefrontal cortex (PFC), is now well established (Goldman-Rakic, 1992). Despite the scope of the behavioral effects of dopamine, knowledge of how PFC neuronal activity is regulated by the mesocortical dopamine system is still limited. Pharmacological experiments in vitro (Cepeda et al., 1992) and in vivo (Williams and GoldmanRakic, 1995; Jedema and Moghaddam, 1996) suggest that the primary action of dopamine transmission in the cortex is modulation of excitatory input on principal neurons. However, there is as yet no consensus on the exact mechanism(s) involved. Although some authors report a mild excitatory effect of dopamine on pyramidal and/or GABAergic neurons of rat prefrontal cortex (Penit-Soria et al., 1987), others have observed inhibitory effects (Law-Theo et al., 1994; Geijo-Barrientos and Pastore, 1995). Yang and Seamans (1996) have suggested that dopamine has differential modulatory effects on pyramidal neurons, depending on synaptic input characteristics, rather than on effects of pure excitation or inhibition.

Anatomical studies have elucidated a possible neural substrate for dopamine-glutamate interaction in the cortex. Dopaminergic axons form an impressive plexus in the PFC of monkeys (Lewis et al., 1987; Goldman-Rakic et al., 1989; Smiley et al., 1992; Williams

Received May 21, 1997; revised July 9, 1997; accepted July 14, 1997.

This work was supported by National Institutes of Health Grants MH 38546, MH 44866, and MH 00298. We thank E. C. Muly III for critical comments on this manuscript and B. Printseva for expert technical assistance.

Correspondence should be addressed to Dr. Leonid S. Krimer, Section of Neurobiology, Yale University School of Medicine, Sterling Hall of Medicine Building, Room C-307, 333 Cedar Street, New Haven, CT 06510.

Copyright (C) 1997 Society for Neuroscience $0270-6474 / 97 / 177450-12 \$ 05.00 / 0$ and Goldman-Rakic, 1993) and humans (Gaspar et al., 1989; Goldman-Rakic et al., 1992). Symmetric dopaminergic synapses are present on spines of pyramidal neurons (Goldman-Rakic et al., 1989; Smiley et al., 1992; Smiley and Goldman-Rakic, 1993), often forming synaptic triads with excitatory inputs on the same spines (Goldman-Rakic et al., 1989; Carr and Sesack, 1996). Dopaminergic axons also have been localized on the dendrites of nonpyramidal cells (Smiley and Goldman-Rakic, 1993; Sesack et al., 1995).

Although all of these studies have revealed new features of the synaptic organization of cortical circuitry involving dopamine, present anatomical approaches have not yet provided a complete view of the total dopaminergic innervation of a given neuron nor addressed whether the location of a neuron within the cortical strata determines the numerical strength of its dopaminergic input. To begin to address these questions, we have combined the technique of intracellular injection of identified neurons in fixed slices with immunohistochemistry for recognition of catecholaminergic sites of contact on the injected cells. Individual pyramidal and nonpyramidal cells of monkey PFC were filled with Lucifer yellow (LY) and subsequently were double-labeled with anti-LY antibody to reveal neurons and with anti-tyrosine hydroxylase $(\mathrm{TH})$ antisera to reveal catecholaminergic axons. The identified neurons and their $\mathrm{TH}$ axonal innervation were reconstructed three-dimensionally, allowing for density and total number of possible synaptic contacts per neuron to be analyzed quantitatively and for comparisons to be made between pyramidal and nonpyramidal cells as well as among pyramidal cells of the different cortical layers. Correlated light and electron microscopy (EM) of the sections containing TH-immunoreactive fibers 
and a LY-filled pyramidal cells was performed to verify whether and in what proportion the TH/LY-labeled contacts identified in the light microscope correspond to close membrane appositions.

\section{MATERIALS AND METHODS}

Tissue acquisition and intracellular injection procedure. Tissue was obtained from five adult rhesus monkeys (Macaca mulata) used in other anatomical experiments. One of the monkeys was used specifically for EM analysis. The monkeys were anesthetized deeply and perfused transcardially with $4 \%$ paraformaldehyde alone or with a mixture of $0.1-0.2 \%$ glutaraldehyde $/ 15 \%$ picric acid in $0.1 \mathrm{M}$ phosphate buffer $(\mathrm{PB})$ $\mathrm{pH}$ 7.4. The brains were removed carefully, and an $\sim 8$-mm-thick single coronal block through the dorsomedial PFC (Brodmann's area 9m) was sectioned immediately into $400 \mu \mathrm{m}$ slices on a vibratome. These slices were held in ice-cold $0.1 \mathrm{M} \mathrm{PB}$ up to $24-48 \mathrm{hr}$ for intracellular injections The method of LY injections was a modification of previously published procedures used in fixed brain slice preparations (Einstein, 1988; Buhl et al., 1990). Penetrating electrodes were pulled from standard borosilicate capillary glass with filament (1 mm outer diameter/ $0.5 \mathrm{~mm}$ inner diameter) on a Flaming Brown horizontal puller (Model P 80/PC, Sutter Instruments, Novato, CA). Electrodes had short $6 \mathrm{~mm}$ shafts (to provide more rigidity while advancing electrodes) and 50-60 $\mathrm{M} \Omega$ resistance. They were back-filled with a $7.5 \%$ solution of LY (dilithium salt, Sigma, St. Louis, MO) in $1 \mathrm{M} \mathrm{LiCl}$. In contrast to traditional methods that use prestaining of slices with fluorescent dyes and that target them under fluorescent illumination, we visualized and injected neurons, using highresolution infrared DIC videomicroscopy typically applied to living brain slices (MacVicar, 1984; Dodt and Zieglgänsberger, 1990, 1994). Each slice was placed in a perfusion chamber with a coverslip affixed to the stage of a Zeiss Axioscop FS microscope (Oberkochen, Germany) and submerged in $0.1 \mathrm{M}$ chilled $\mathrm{PB}$. With this approach [using a highresolution water-immersed $63 \times$ objective with numerical aperture (N.A.) of 0.9 and infrared DIC videomicroscopy equipped with a $4 \times$ magnification tube (Stuart et al., 1993)], all parts of the neurons-their apical dendrites, cell bodies, nuclei, and nucleoli as well as the microelectrode tip - could be visualized with high resolution (see Fig. 1). Under visual control, pyramidal and nonpyramidal neurons in different cortical layers were injected selectively with LY at a depth of 30-50 $\mu \mathrm{m}$ from the tissue surface. Pyramidal cells were distinguished from interneurons by their larger size and the presence of apical dendrites. Glass microelectrodes were advanced through the tissue while a "holding" constant positive current $0.2-0.5 \mathrm{nA}$ was applied (depending on electrode resistance) to avoid LY labeling of nonrelated structures. To prevent electrode plugging caused by extensive positive current, we adjusted the holding current to be just large enough to block LY diff using from the electrode tip. Electrodes were positioned precisely inside the neuronal nucleus (see Fig. 1). To assure membrane penetration and prevent staining of adjacent axons or dendrites, we briefly applied the capacitance overcompensation mode. Cells were filled with LY either by diffusion (in weakly fixed tissue) or by applying $0.3-0.4 \mathrm{nA}$ constant negative current for 15-25 min (or a shorter time for smaller cells). Injections were terminated when bright fluorescence was observed in the distal dendrites and spines (in the case of pyramidal cells) (see Fig. 1). Generally, two to four cells were injected per slice, and injections were far enough apart to avoid overlapping. Electrode resistance measurements, current injection, and capacitance overcompensation were provided by an intracellular Neuroprobe amplifier (A-M System, Everett, WA).

Tissue processing and immunohistochemistry. Immediately after injections, slices were post-fixed for 3-4 hr in 4\% paraformaldehyde and then rinsed and left in $0.1 \mathrm{M}$ PB overnight. They were resectioned on a microslicer (DSK, Dosaka, Japan) into 40- $\mu$ m-thick sections. To obtain perfectly aligned slices, we trimmed an agar block attached to the stage of the microslicer to match the blade plane. Then a piece of a glass slide with scratched surfaces was glued to the newly formed agar platform, and slices were affixed flat to the glass by a thin film of cyanoakrylate glue. Six consecutive sections were obtained from each slice, starting from the injected surface. For the double immunohistochemical protocol, sections first were placed in cold blocking serum (10\% normal goat serum, $2 \%$ BSA, and $0.5 \%$ Triton X-100) containing either avidin (for the first 30 $\mathrm{mm}$ ) or biotin (for another $30 \mathrm{~min}$ ). Avidin and biotin were obtained from Vector's AB blocking kit (Burlingame, CA). After a brief rinsing, sections were incubated in a mixture of rabbit biotinilated anti-LY (Molecular Probes, Eugene, OR) and mouse anti-TH (Chemicon, Te- mecula, CA) primary antisera (diluted 1:500 and 1:1000, respectively) for $48 \mathrm{hr}$ at $4^{\circ} \mathrm{C}$. Next the sections were incubated in the ABC Elite reagent (1:50 dilution) for $1 \mathrm{hr}$ at room temperature, and the reaction was developed with the brown chromogen $3^{\prime}, 3^{\prime}$ diaminobenzidine (DAB), using the glucose oxidase method. Under direct microscopic control, neurons were developed until a barely visible background stain appeared. Then $0.3 \% \mathrm{H}_{2} \mathrm{O}_{2}$ in $\mathrm{PB}$ was applied for 20 min to block the residual peroxidase activity on the labeled neurons. Sections were rinsed and incubated in anti-mouse IgG (diluted 1:200 in blocking serum) for $1.5 \mathrm{hr}$. Again the ABC Elite reagent was applied, but, in this instance, Nickel intensification of the $\mathrm{DAB}$ reaction $(\mathrm{Ni}-\mathrm{DAB})$ was used to obtain a blue-black staining of $\mathrm{TH}$ axons. To diminish shrinkage of section thickness, they were not air-dried on gelatin-coated slides but dehydrated while free-floating. During the dehydration procedure, sections were covered with filter paper in low concentrations of alcohol. After being cleared in xylene, the sections were mounted on slides with Cytoseal and coverslipped. To estimate neuronal shrinkage during the dehydration procedure, we traced two basal dendrites of an LY-injected pyramidal cell on the Neurolucida under fluorescence at $400 \times$ before dehydration and then again after dehydration and clearing in xylene. Histological processing resulted in a $30 \%$ reduction in total dendritic length (370 vs $259 \mu \mathrm{m})$. The data are presented both as direct measures and as corrected values, the latter in parentheses, after the direct measures.

For EM, a two-cycle freeze-thaw procedure was applied to improve antibody penetration, because treatment of sections with detergent (Triton $\mathrm{X}-100)$ disrupts the lipid membranes. The $400 \mu \mathrm{m}$ slices containing the LY-injected cells were placed in $15 \%$ sucrose for $3 \mathrm{hr}$, snap-frozen in liquid nitrogen (twice), and thawed gradually. Then the slices were resectioned at $40 \mu \mathrm{m}$ and processed according to the light microscopic immunostaining protocol described above, but without the addition of Triton X-100 to the incubating solutions. TH-immunoreactive boutons were visualized with the chromogen Ni-DAB, and the LYimmunolabeled dendrites were stained with the chromogen DAB. These two chromogens were chosen for EM because their dense, dark reaction product was necessary to recognize and unambiguously identify the labeled structures under the light microscope in the dark-colored resinembedded sections. Aided by this technique, we were able to detect TH-immunolabeled fibers and dendrites of the LY-labeled layer III pyramid up to $\sim 8 \mu \mathrm{m}$ deep in the tissue. After visualization of the antigens, the sections were osmicated in $1 \% \mathrm{OsO}_{4}$, dehydrated, and flat-embedded in Durcupan resin.

Data analysis. Pyramidal neurons with TH appositions were reconstructed three-dimensionally using Neurolucida software and a hardware package (Microbrightfield) mounted on an Axioskop Zeiss microscope. This software yields morphometric values for dendritic lengths and soma size (outlined at its largest dimension). It also allows TH appositions to be marked on the traced dendritic tree as well as to calculate the number and density of these contacts. Microscopic analysis was conducted with a high resolution $63 \times$ oil objective (1.4 N.A.) and DIC contrast enhancement, oil condenser (1.4 N.A.), and two additional interimage lenses $(2 \times$ and $1.25 \times$ ), which together yielded a final magnification of $1575 \times$ magnification. Sections were counterstained with Nissl stain only after the three-dimensional analysis was completed for delineation of the laminar borders. Identified neurons were assigned to cortical layers by location of their soma.

TH axonal contacts on LY-labeled processes were observed readily. Only TH axons that made direct physical contacts with dendritic spines or shafts were considered appositions. The absence of any measurable space between the TH-labeled process and the target dendrites was adopted as the criterion for apposition and was applied strictly. In situations in which a single dopaminergic axon obliquely crossed several spines and a dendritic shaft and could have, in fact, more than one apposition, to be conservative it arbitrarily was counted as one apposition. Data also were collected on section thickness. Accurate $z$-axis values were displayed automatically on the computer screen while focus was changed from the top to the bottom of the sections. For EM analysis, portions of the spiny dendritic tree were selected under the light microscope and then traced with a Neurolucida program. Four close appositions between TH fibers and labeled dendrites were marked on the traced dendrites and also were photographed. The selected dendritic segments in an $\sim 8-\mu$ m-thick superficial layer of the tissue sections were reembedded in blocks and serially cut. Thin sections (150) were collected on Formvar-coated single-slot grids and analyzed with a JOEL 100 electron microscope.

Means and SD for TH apposition density (THAD) were obtained for 
each cell grouping, and statistical comparisons were made by unpaired $t$-tests. In addition, the nonparametric Mann-Whitney $U$ test was used for analysis of layer II-IIIs pyramids versus layer III pyramids.

\section{RESULTS}

Both pyramidal and nonpyramidal neurons were filled well with LY and recovered with the same quality after immunostaining (Figs. 1, 2A-E ). Dendrites studded with numerous spines (Fig. $2 A$, inset) were, for the most part, visualized completely to the end of their distal branches. The only exceptions were the distal branches in layers I and II of apical dendrites originating from some neurons in deeper layers, i.e., from deep layer 3 and below, which were not always filled completely with LY and therefore were not fully recovered. Well-labeled TH-immunopositive axons were observed through layers 1-3 and 5-6, with their greatest elaboration in layers 1-2, (Fig. 2A-E). An important technical feature is that each individual $\mathrm{TH}$ axon or neuronal dendrite could be followed throughout the section thickness, indicating complete antibody penetration.

\section{Pyramidal neurons}

A total of 19 pyramidal cells was analyzed. The laminar distribution of principal cells was as follows: two cells from layer II, nine from layer III, four from layer V, and four from layer VI (Table 1). After analysis was completed, the one neuron located superficially in layer III and two cells of layer II were regrouped in one category renamed layer II-IIIs (superficial). The reclassification of the layer III cell was based on two features that distinguished this neuron from the neurons located in deeper portions of layer III: (1) the soma of this neuron resided in the uppermost portion of layer III (based on Nissl counterstain), with many of its dendrites embedded in the dense horizontal band of $\mathrm{TH}$ axons that is characteristic of layers I-IIIs (Williams and Goldman-Rakic, 1993); (2) the main shaft of its apical dendrite was comparatively short with a rich lateral arborization. Completely filled neurons were reconstructed from six consecutive 40 $\mu \mathrm{m}$ sections. However, because injections of cells were made at $40-50 \mu \mathrm{m}$ from the cut surface of the slice, approximately onethird of the dendritic arbor extending beyond the surface of the section generally was lost (see Fig. 6b). All reconstructions, therefore, comprised two-thirds of the arbor of each pyramidal cell. The mean total dendritic length was $8.3 \mathrm{~mm}(10.8 \mathrm{~mm})$ per pyramidal neuron (calculated with the exception of the smallest layer II neuron) (Table 1). TH labeled axons were very tortuous and showed complex morphological relationships with dendrites of pyramidal cells. They often approached the dendrites with a shallow angle, first contacting the spine head, then passing in the vicinity of several spine necks (or contacting them), and, finally, crossing the dendritic shaft (Fig. $3 f$ ). They could coil around the dendrite or travel along dendrites from several to $50 \mu \mathrm{m}$ on one occasion). In contrast to the dendrites in layers III-VI, the distal branches of apical dendrites in layer I-II usually were crossed simply by $\mathrm{TH}$ axons at right or close to right angles.

The TH axonal appositions on pyramidal cells were relatively evenly distributed, particularly on the secondary and more distal branches although generally avoiding the primary basal or apical dendrites (see Fig. 6). The one layer III pyramidal neuron with the most completely reconstructed apical dendrite distal branches (see Fig. 6) had virtually the same THAD on its apical (0.72) and its basal (0.79) dendrites. Appositions were observed on both dendritic spines and shafts (Fig. $3 e-h$ ) and appeared to be distributed evenly between these two structures. The mean THAD

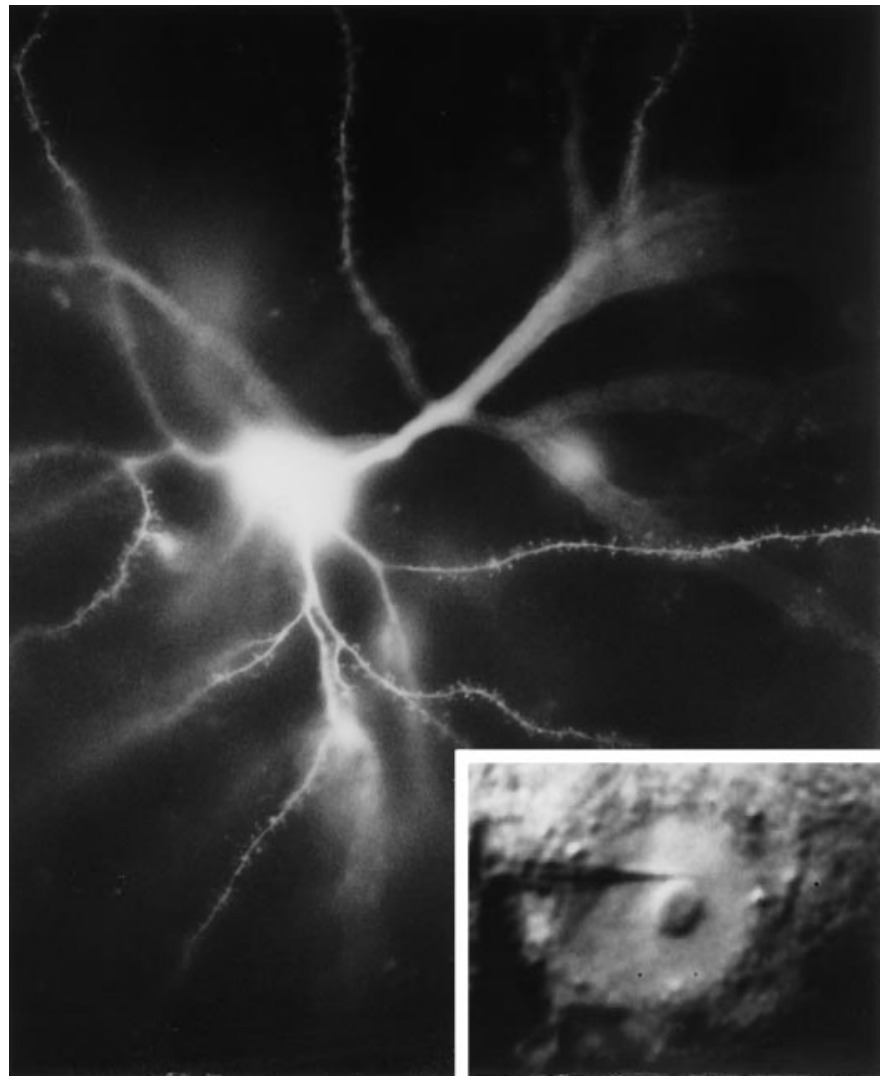

Figure 1. Lucifer yellow injection of pyramidal neurons in fixed slices. On the inset, the glass microelectrode filled with LY positioned inside the cell nucleus is under visual control provided by DIC-infrared videomicroscopy; loaded with LY, the neuron is observed with fluorescence microscopy (note numerous spines on the dendrites).

per $100 \mu \mathrm{m}$ of dendritic length was $0.7 \pm 0.04(0.5 \pm 0.04)$ for pyramidal neurons of layers III-VI, and the average total number of appositions per reconstructed pyramidal neuron was 60 (Table $1)$. Values of the mean density were the same on principal cells of different layers except for layer II-IIIs (Table 1). The number of appositions per unit length of the spiny dendrites in this layer was significantly higher ( $p<0.0003$ by $t$ test; $p<0.01$ by MannWhitney $U$ test) and, in fact, double that of any other layer $[1.47 \pm$ $0.2(1.04 \pm 0.2)]$ (Table 2). This finding is in agreement with the noticeably higher $\mathrm{TH}$ axonal density in this lamina observed in the present study and previously in the literature (Lewis et al., 1988; Williams and Goldman-Rakic, 1993). Interestingly, the variance in the THAD among the pyramids within a given cortical layer was relatively low [coefficient of variance $(\mathrm{CV})=11-20$ ] as was the variance among the different cortical layers, excepting layer II-IIIs $(\mathrm{CV}=16)$.

EM examination recovered all four $\mathrm{TH} / \mathrm{LY}$ contacts that were recognized in the light microscope (Figs. 4, 5) and confirmed that the contacts between the labeled profiles represent close membrane appositions. As shown (Fig. $4 A_{2}-A_{5}, B_{2}$ ), the plasma membranes of TH-immunoreactive axons (labeled with Ni-DAB) indeed were closely apposed to the plasma membranes of the LY-immunopositive dendrites (labeled with DAB), and these appositions could be followed through in serial sections (Fig. 


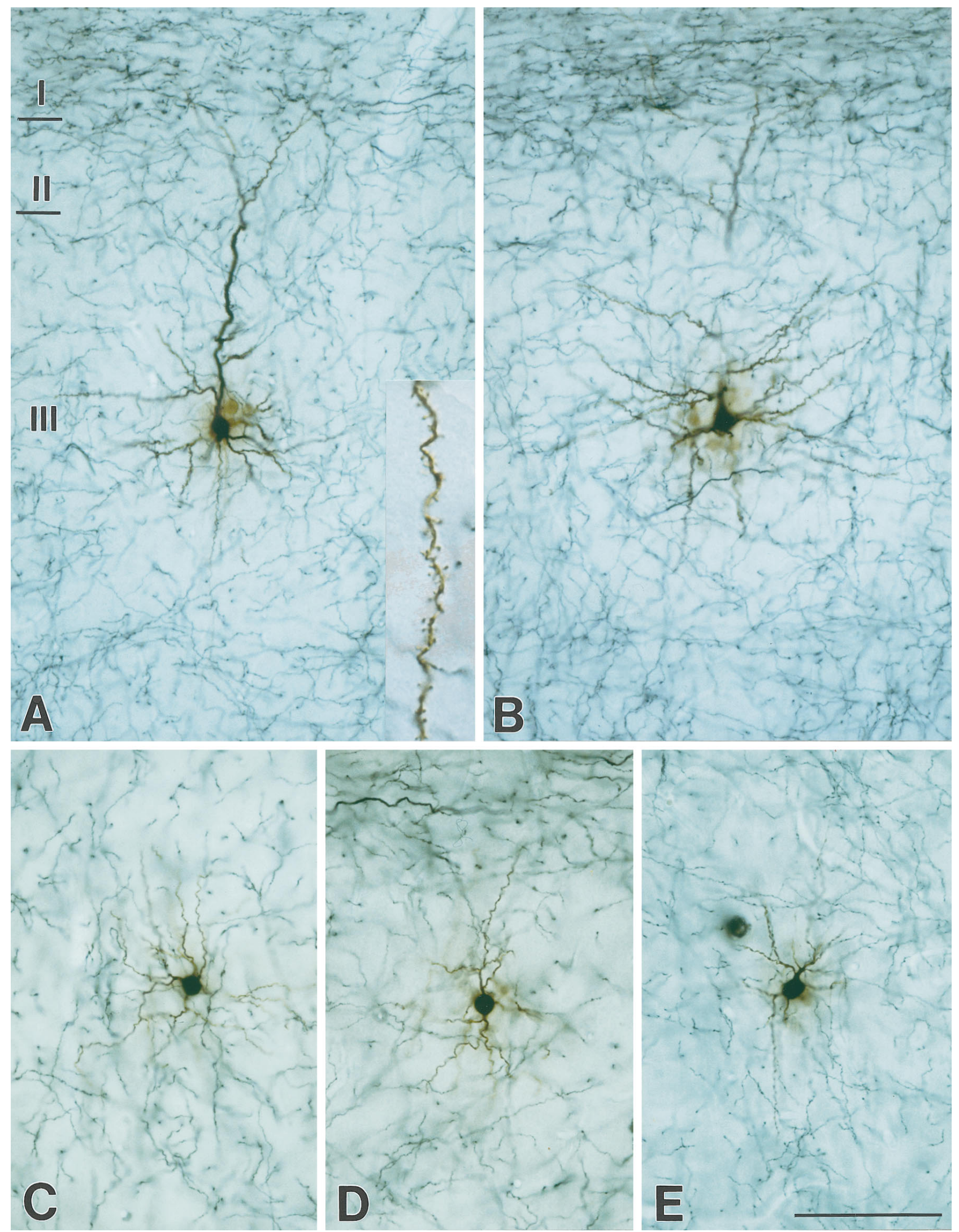

Figure 2. Low-power photographs of double-immunostained cortical pyramidal/nonpyramidal neurons and TH axons. $A$, $B$, Two consecutive sections of the same pyramidal neuron. $C-E$, Nonpyramidal neurons. Scale bar, $100 \mu \mathrm{m}$. 

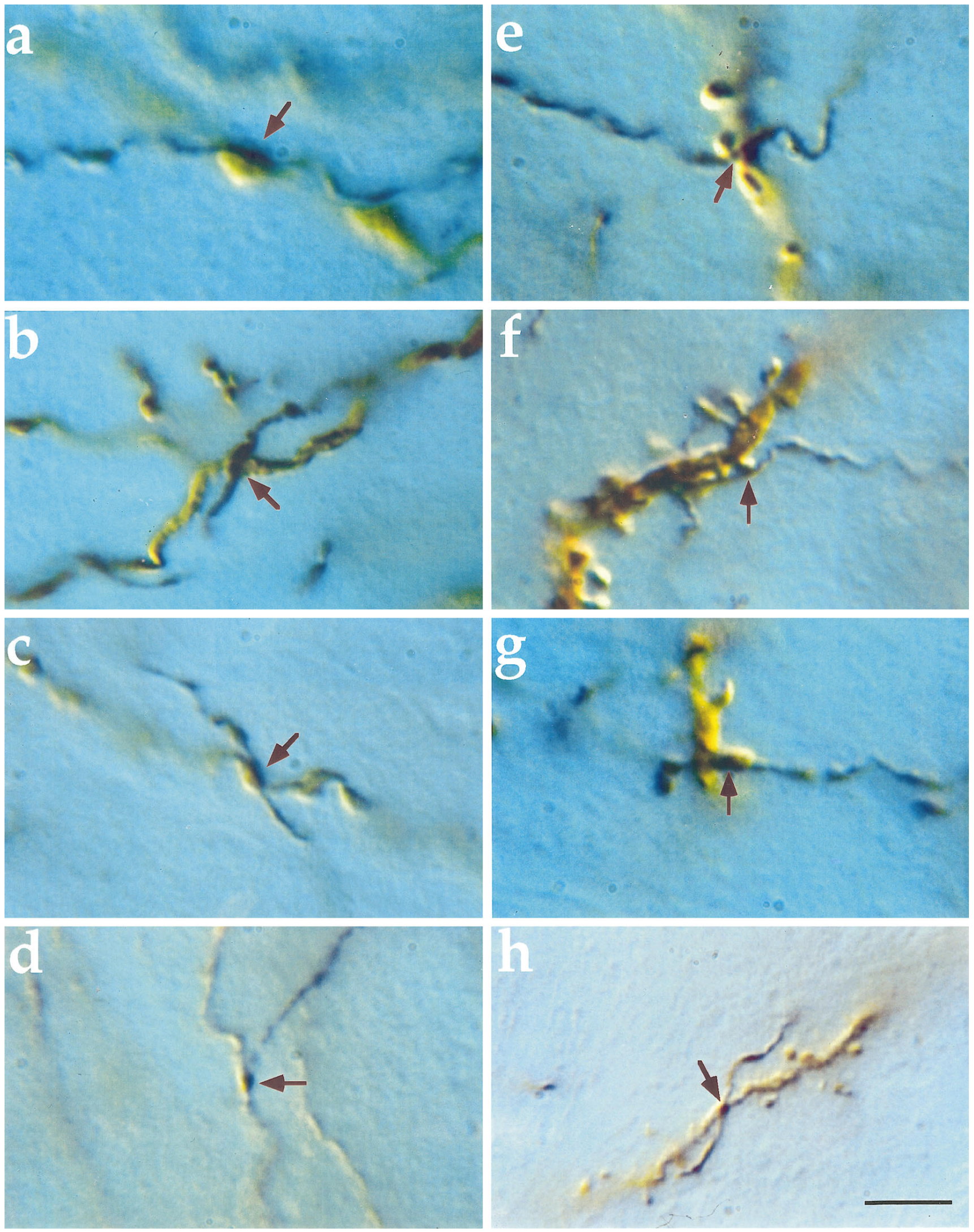

Figure 3. High-power photographs of TH appositions to the neuronal dendrites. $a-d$, Appositions to the smooth dendrites (indicated with arrows). $e-h$, Appositions to spiny dendrites; $e$, to neck of spine; $f$, $g$, to head of spine; $h$, to shaft. Scale bar, $5 \mu \mathrm{m}$. 

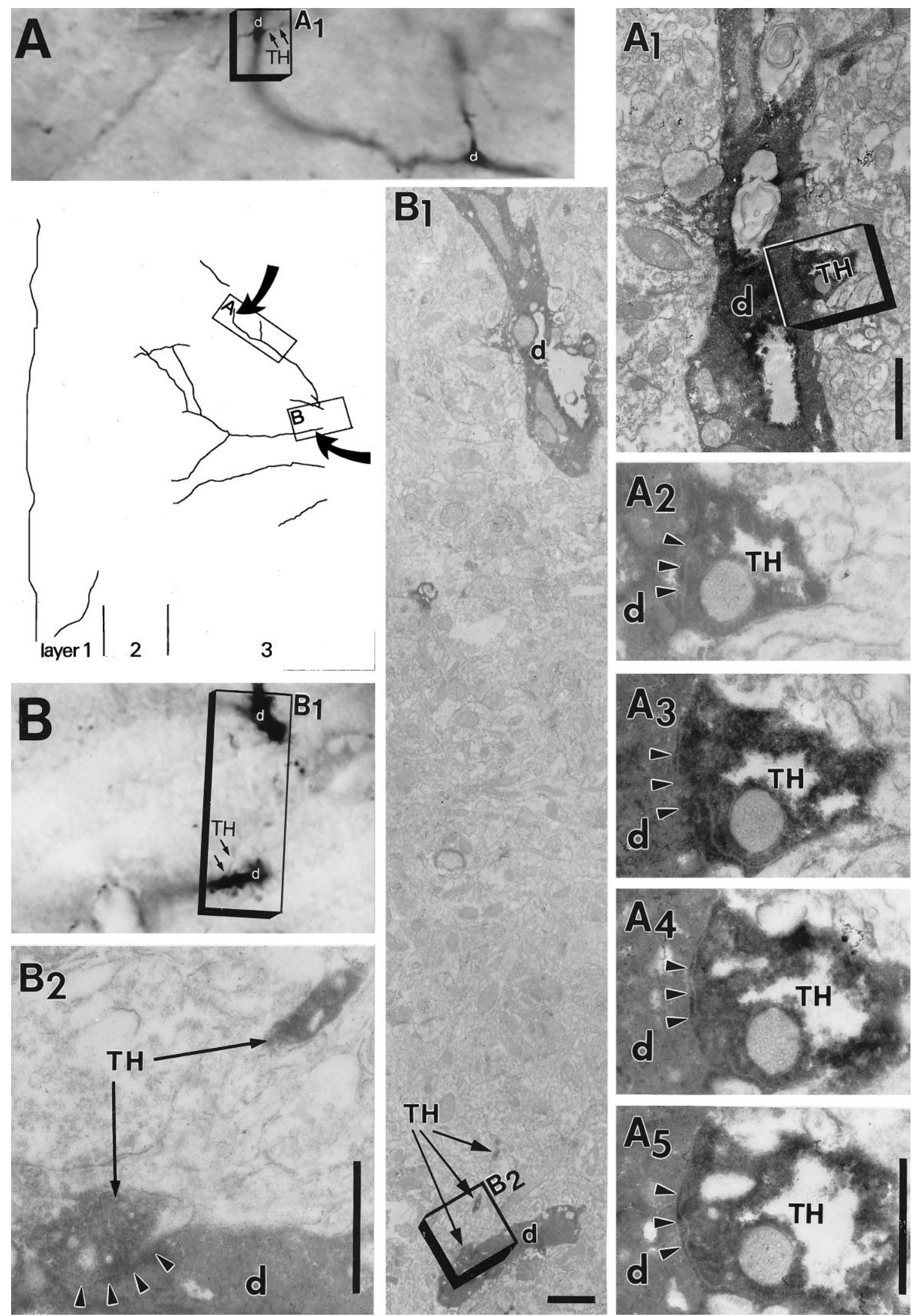

Figure 4. Correlated light and electron microscopic analysis of a 40- $\mu \mathrm{m}$-thick section containing TH-immonolabeled fibers and the dendritic arbor of a pyramidal neuron intracellularly filled with LY. The analyzed area contains cortical layers I-III. The line drawing shows the dendritic arbor that was traced with the Neurolucida tracing system. The boxed areas in $A$ and $B$ depict two sites on the dendritic arbor in which a TH-labeled fiber is closely apposed (arrows) to a dendritic shaft. $A$ (above the line drawing) and $B$ (below the line drawing) are high-power light micrographs of these two boxed areas showing the two dendritic shafts that are each contacted by a TH-immunolabeled fiber (TH; arrows). The framed areas $A_{1}$ and $B_{1}$ of the light micrographs are shown enlarged on electron micrographs $A_{1}$ and $B_{1}$, each depicting a TH-labeled bouton (TH) in close apposition with a LY-filled dendritic shaft $(d)$. These two TH boutons contacting the labeled dendrites are shown further enlarged on high-power electronmicrographs in $A_{2}-A_{5}$ and $B_{2}$, respectively. Arrowheads point to the close apposition between the membranes of the TH-labeled axonal and LY-labeled dendritic profiles. Scale bars: $A_{1}, B_{1}, 1 \mu \mathrm{m} ; A_{2}-A_{5}, B_{2}, 0.5 \mu \mathrm{m}$. 


\begin{tabular}{lllll}
\hline Table 1. TH apposition density (THAD) on pyramidal neurons in different cortical laminae & & \\
& Mean THAD (per 100 & $\begin{array}{l}\text { Total number } \\
\text { of appositions }\end{array}$ & $\begin{array}{l}\text { Total dendritic } \\
\text { length (in mm) }\end{array}$ & $\begin{array}{l}\text { Soma size } \\
\text { (in } \mu \mathrm{m})\end{array}$ \\
Cortical laminae & $\mu$ m of dendritic length) & $\begin{array}{l}\text { Number of } \\
\text { analyzed neurons }\end{array}$ \\
\hline II & $1.65 \pm 0.21$ & $58 \pm 23$ & $3.7 \pm 1.9$ & $46.7 \pm 8.8$ \\
IIIs & 1.1 & 119 & 10.7 & 62.4 \\
IIId & $0.72 \pm 0.04$ & $64 \pm 5.0$ & $8.9 \pm 0.4$ & $54.9 \pm 1.9$ \\
V & $0.73 \pm 0.04$ & $56 \pm 4.0$ & $7.6 \pm 0.1$ & $60.7 \pm 1.6$ \\
VI & $0.73 \pm 0.04$ & $57 \pm 7.0$ & $7.9 \pm 1.2$ & $59.7 \pm 3.6$ \\
Deeper III-VI & $0.71 \pm 0.03$ & $60 \pm 3.0$ & $8.3 \pm 0.4$ & $57.6 \pm 1.4$ \\
\hline
\end{tabular}

s, Superficial; d, deep.

Table 2. Comparison of THAD on pyramidal versus nonpyramidal neurons in layer III and in superficial layers (II + IIIs) versus deep layer III

\begin{tabular}{llll} 
Neuronal type & Layer III & Layers II + IIIs & Layer IIId \\
\hline Pyramidal & $0.76 \pm 0.17^{*}$ & $1.47 \pm 0.20^{* *}$ & $0.72 \pm 0.04$ \\
& $(n=9)$ & $(n=3)$ & $(n=8)$ \\
Nonpyramidal & $0.43 \pm 0.05$ & $0.53 \pm 0.07$ & $0.40 \pm 0.06$ \\
& $(n=17)$ & $(n=4)$ & $(n=13)$ \\
\hline
\end{tabular}

*Significantly higher THAD on pyramidal versus nonpyramidal neurons in layer III ( $p<0.0003$ by unpaired $t$ test); **significantly higher THAD on pyramidal neurons in superficial layers versus deep layer III ( $p<0.0003$ by unpaired $t$ test; $p<0.01$ by Mann-Whitney $U$ test); s, superficial; d, deep. $\left.4 A_{2}-A_{5}\right)$. TH appositions on labeled spines are shown in Figure 5, $A$ and $B$; both appositions are to the necks of spines (one at the confluence of the neck with the parent dendrite). Unfortunately, the double-label approach necessary to examine these appositions on identified cells precludes definitive analysis of their synaptic/ nonsynaptic nature. However, the stronger labeling of dendritic membranes apposed to the TH boutons (Fig. $4 A_{2}, B_{2}$ ) might suggest the presence of synaptic specializations.

\section{Nonpyramidal neurons}

Seventeen interneurons were analyzed in the study, all from layer III. Because of their smaller size and the predominantly radial
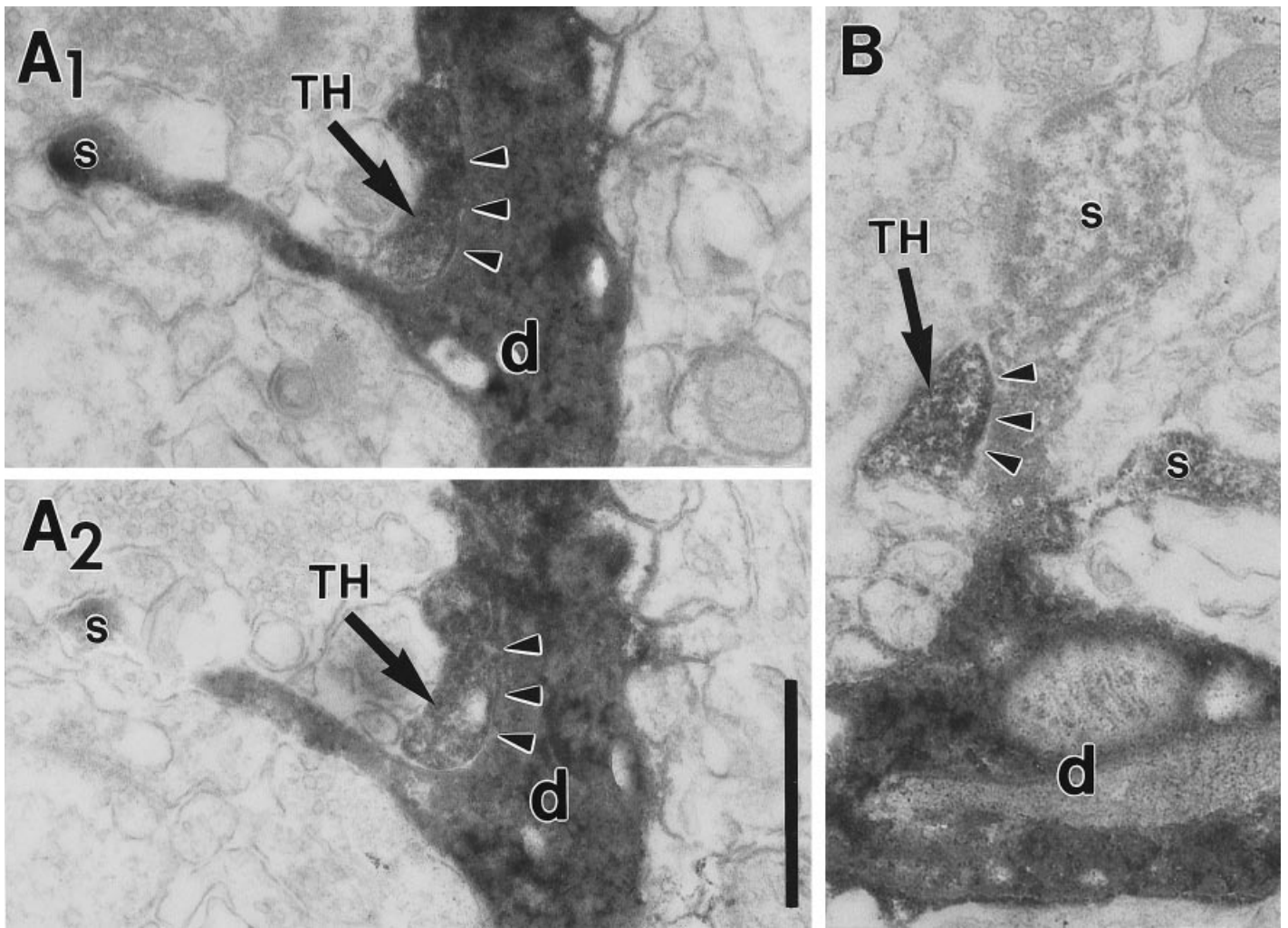

Figure 5. Electron micrographs $\left(A_{1}, A_{2}, B\right)$ portraying two spiny dendrites $(d)$ of a Lucifer yellow-filled pyramidal cell, each contacted by a TH-immunoreactive axon. The TH-positive axons are oriented toward the base $\left(A_{1}, A_{2}\right)$ or neck $(B)$ of the dendritic spine $(s)$. The closely apposing membranes of the labeled structures are indicated by arrowheads. Scale bar, $0.5 \mu \mathrm{m}$. 
$\mathbf{a}$

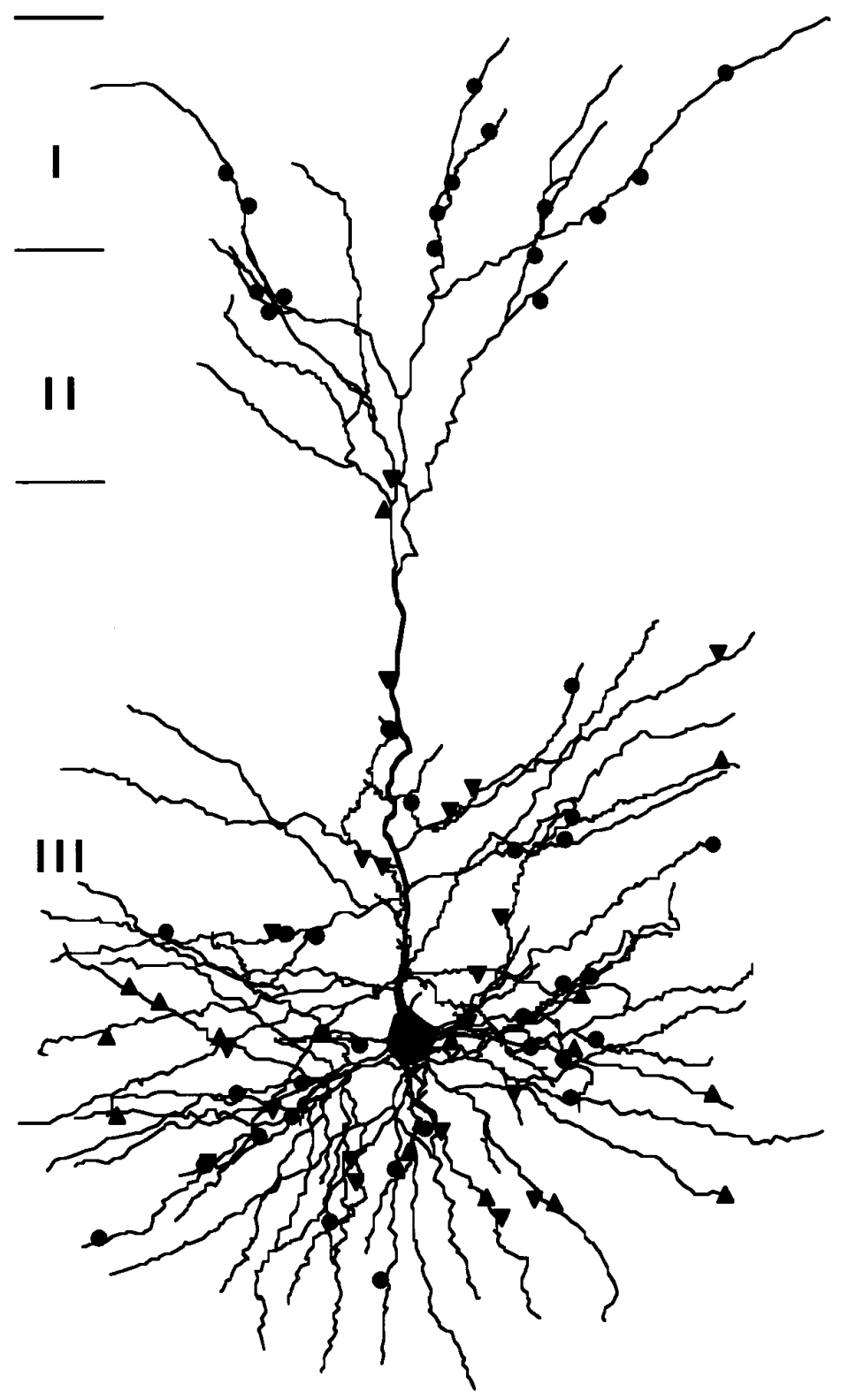

b

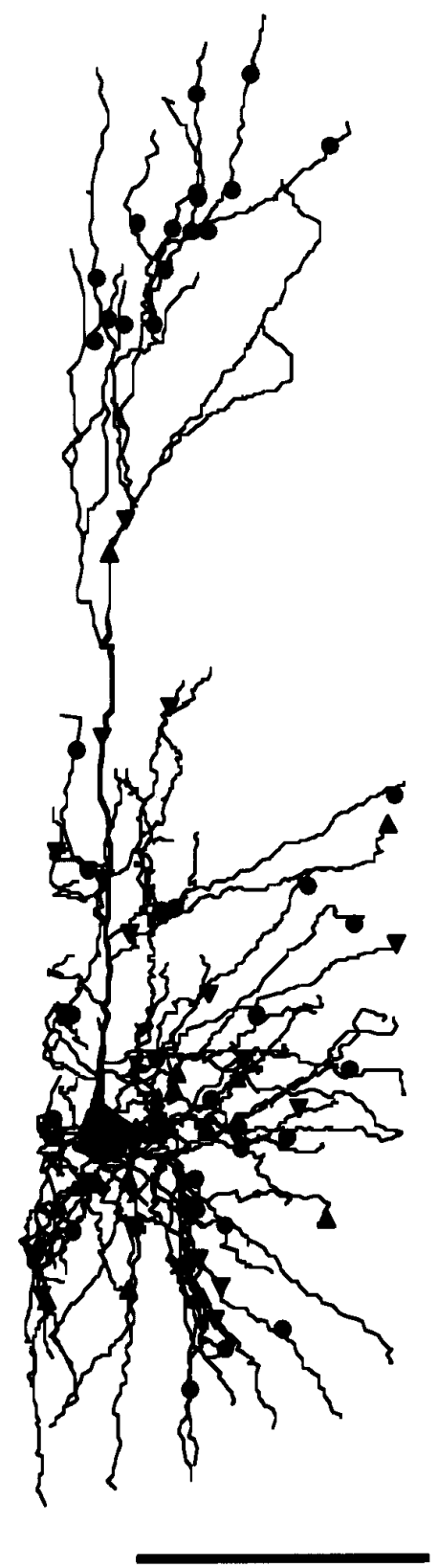

Figure 6. Three-dimensional reconstructed pyramidal cell with TH appositions. $a$, The entire neuron was reconstructed from the six separate drawings. $b$, The neuron is rotated $90^{\circ}$ along the $y$-axis. $\mathbf{\Delta}$, Appositions to spines; $\boldsymbol{\bullet}$, appositions to shafts. Scale bar, $100 \mu \mathrm{m}$.

orientation of their dendritic arbors, reconstructions could be made in as few as 1, 2, or 3 sections. TH appositions to dendrites often were observed (Figs. $3 a-d, 7)$. Nonpyramidal cells had more clear-cut $\mathrm{TH}$ crossings than pyramidal neurons, and $\mathrm{TH}$ axons usually did not follow along dendrites. The average THAD on interneurons was nearly one-half that on pyramidal cells $(p<$ $0.0003)$ and comprised only $0.4 \pm 0.05(0.3 \pm 0.05)$ per $100 \mu \mathrm{m}$ of dendritic length (Table 2). Four nonpyramidal cells belonged to layer IIIs and, similar to the pyramidal cells of this sublamina, showed a trend to higher THAD (although not significant, $p<$ $0.2)$ than the rest of the layer III nonpyramids $[0.53 \pm 0.07$ vs
$0.4 \pm 0.05$, respectively $(0.37 \pm 0.07$ vs $0.3 \pm 0.05)$ (Table 2$)]$. In striking contrast with pyramidal cells, we found a high variance of THAD value among the interneurons $(\mathrm{CV}=45)$ with a range of $0.1-0.8$ contacts per $100 \mu \mathrm{m}$ (see Fig. 8). Some interneurons had only a single TH apposition, whereas others had as many as an average pyramidal cell of layer III, raising the possibility that different subtypes of interneurons may receive different numbers of $\mathrm{TH}$ contacts. Also in contrast to pyramidal neurons, potential contacts here often were distributed unevenly and sometimes could be found only on one pole of a dendritic arbor (Fig. 7). 
Figure 7. Three-dimensional reconstructed nonpyramidal cells with $\mathrm{TH}$ appositions. $\mathbf{0}$, Appositions to shafts. Scale bar, $100 \mu \mathrm{m}$.

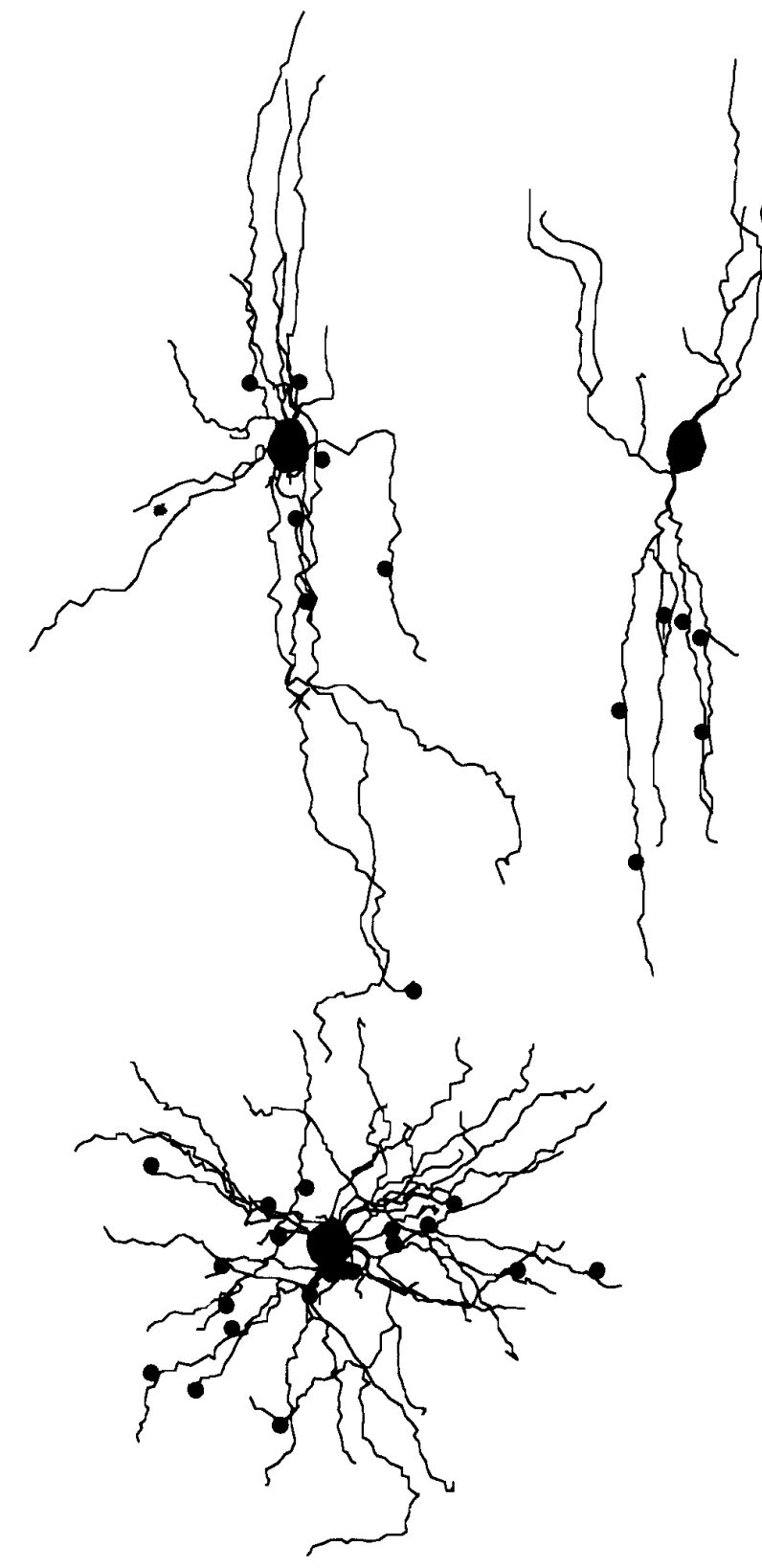

\section{DISCUSSION}

The present study provides a complete morphometric threedimensional reconstruction of prefrontal cortical neurons targeted by catecholaminergic axons (labeled here by $\mathrm{TH}$ ) with measurements of the total number as well as the density of TH appositions per neuron. The methodology applied has yielded new quantitative information and direct comparisons of the distribution of possible dopaminergic terminals among pyramidal/nonpyramidal cells and pyramids of different layers. Accurate quantitative assessment of the catecholaminergic innervation of the cortex now can be used in comparative studies of brain development, aging, and human and experimental neuropathology.

\section{Methodological issues}

The combination of a fixed slice preparation and double immunohistochemistry yielded high-quality labeling of selected neurons, complete antibody penetration, and clear chromatic distinctions of fine neuronal structures such as spines (soft brown label) and thin TH axons (stained in blue-black). These features are critical for a quantitative analysis of synaptic input on identified neurons. Another important methodological feature was the preservation of the $z$-axis dimension of the sections during the dehydration procedure. Close appositions in that axis are particularly difficult to analyze, and extensive shrinkage of sections can produce artifactual contacts by bringing distant structures into apparent close appositions. Our free-floating dehydration procedure 


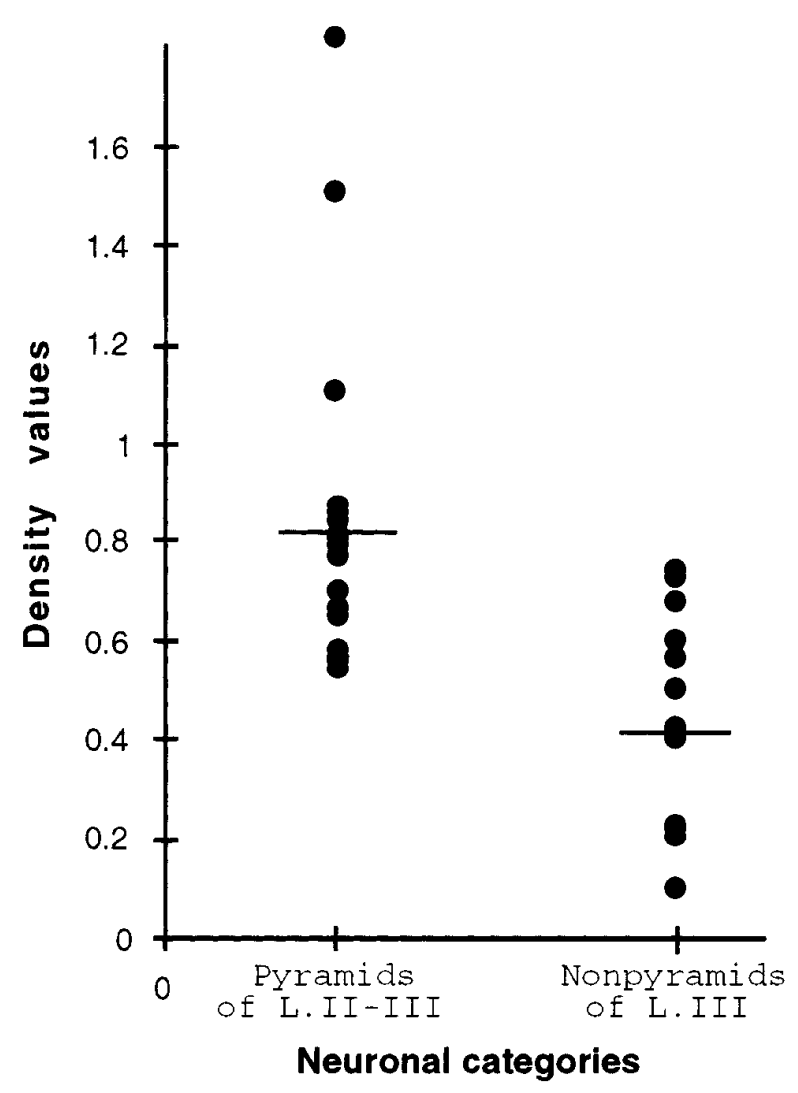

Figure 8. Comparative distribution of THAD on pyramidal and nonpyramidal cells in layers II and III. Pyramids have a statistically significant twofold higher mean THAD than nonpyramids (0.8 vs $0.4 / 100 \mu \mathrm{m}$ of dendritic length; $p<0.0003$ ). With the exception of the three highest values for pyramids of the layer II-IIIs category, the variance of THAD in pyramids is one-half that of nonpyramids. Nonpyramids exhibit a wide range of THADs from 0.1 to $0.8 . \mu \mathrm{m}$.

causes only $35 \%$ shrinkage of the sections in the $z$-axis (final thickness of $40 \mu \mathrm{m}$ cut sections was $26 \mu \mathrm{m}$ ), whereas traditional methods, by direct comparison, reduce section thickness by $75 \%$ (sections cut at $40 \mu \mathrm{m}$ shrink to $10 \mu \mathrm{m}$ thickness).

There are also limitations to the present approach. As is well known, TH is not specific for dopamine, and antisera directed to dopamine theoretically would be a better choice. However, the strong fixation with $5 \%$ glutaraldehyde needed to fix the smaller dopamine molecule excludes filling neurons by intracellular injection. On the other hand, several studies have emphasized that anti-TH antisera in the primate cortex, including area $9 \mathrm{~m}$, visualize predominantly dopaminergic axons with, at most, only $1 \%$ among them constituting noradrenergic processes (Lewis et al., 1988; Williams and Goldman-Rakic, 1993; Sesack et al., 1995). Also, direct comparisons of dopamine and $\mathrm{TH}$-immunostained axons in the cortex with light microscopy (Williams and GoldmanRakic, 1993) and their synaptic contacts with EM (Sesack et al., 1995) failed to reveal any significant difference between them.

It is possible that in layer I the uppermost distal branches of pyramidal apical dendrites have a higher THAD than other dendrites. Although our data on this point are limited, we could note that THAD was not higher on the reconstructed apical dendrites, as compared with basal dendrites. Indeed, THAD on completely filled pyramids was similar to neurons with incompletely labeled apical dendrites, and THAD on the apical dendrites (0.72) was remarkably similar to THAD on the basal dendrites (0.79) of the one layer III pyramidal neuron with the most completely reconstructed apical dendrite.

Although the optical setup used in the present study yielded very good visualization of fine neuronal structures, this is obviously far short of EM resolution. Although dopamine synapses are very difficult to analyze with EM [because many axonal varicosities are nonsynaptic (Descarries et al., 1991) and serial reconstruction of $\mathrm{TH}$-immunopositive boutons reveals that only $39 \%$ of them form conventional synaptic appositions (Smiley and Goldman-Rakic, 1993)], we supplement our light microscopic examination with ultrastructural analysis. EM analysis in this particular situation is restricted both by the fact that only one or few identified cells are available per preparation and that poor antibody penetration in thick sections dramatically reduces the opportunity to detect labeled appositions. Nevertheless, the correlated EM analysis of double-labeled material provided further evidence that the contacts identified under the light microscope indeed do correspond to close membrane appositions at the ultrastructural level and therefore may represent functional sites of interaction between the TH-containing fiber system and the pyramidal neurons of the cerebral cortex. The presence of dark chromogens in the axonal and dendritic components contacting each other obscures the unambiguous identification of possible synapses, although some of the contacts seem to have such membrane specializations. As mentioned earlier, previous studies have established that there are dopaminergic synapses on dendritic shafts and spines (Goldman-Rakic et al., 1989; Smiley et al., 1992; Smiley and Goldman-Rakic, 1993).

\section{TH innervation of pyramidal neurons}

Pyramids of layers II and IIIs had significantly higher densities of TH appositions (1.4 per $100 \mu \mathrm{m}$ of dendritic length), as compared with pyramids in the other layers, in keeping with previous immunohistochemical studies (Lewis et al., 1987; Williams and Goldman-Rakic, 1993). Reconstructed pyramidal cells residing within layers III, V, and VI exhibited the same mean THAD of 0.7 per $100 \mu \mathrm{m}$ of dendritic length, and this parameter exhibited low variance $(\mathrm{CV}=16)$. This finding suggests that all pyramidal neurons in area $9 \mathrm{~m}$ may receive a dopaminergic input of consistent apposition density, independent of laminar positioning in the cortex. This remarkable uniformity also was evident in the distribution of appositions along spiny dendrites (avoiding, however, primary dendrites). Because a single basal dendrite of a layer III neuron (measured from the cell body to the distal end) is $\sim 150$ $\mu \mathrm{m}$ in length, each basal dendrite would have, on average, one TH apposition.

The total number of TH appositions per reconstructed neurons in these laminae averaged 63 . Considering that one-third of the pyramidal dendritic tree was not recovered, by extrapolation we estimate that an entire pyramidal cell in these layers receives, on average, 90 contacts. It may seem that this number of contacts, by itself, is not enough to explain the powerful modulatory effect of dopamine on excitatory neurotransmission in cortical neurons, particularly in light of the enormous number of dendritic spines occupied by asymmetric synapses. However, recent evidence from single neuron recordings in awake monkey PFC shows that iontophoretic application of dopaminergic agents produces strong effects on their neuronal firing properties (Sawaguchi and Goldman-Rakic, 1994; Williams and Goldman-Rakic, 1995), compatible with other evidence that dopamine is a powerful modulator of neuronal activity in cortical circuits. A volume transmission mechanism of dopaminergic modulation is also a strong 
possibility to complement presumed synaptic action (Kawagoe et al., 1992; Garris and Wightman, 1994; Garris et al., 1994), because $\mathrm{TH}$ axons frequently were observed coursing in the vicinity of spiny dendrites for considerable distances (up to $50 \mu \mathrm{m}$ ). The twofold stronger $\mathrm{TH}$ innervation of layer II and IIIs pyramidal cells may provide an additional morphological basis for synaptic interactions. These neurons are likely to perform extensive intrinsic processing functions, which may be particularly important for prefrontal function.

Our results on $\mathrm{TH}$ apposition density of pyramidal neurons may be compared with data on the dopaminergic innervation of rat neostriatum (of Freund et al., 1984). These authors reported $34 \mathrm{TH}$ synaptic terminals on the spines of a $75 \mu \mathrm{m}$ length of a reconstructed dendrite, which represented $62 \%$ of total synapses on spiny dendrites (shaft synapses account for 38\%). Taking these figures into account, we calculate a density of 75 contacts per $100 \mu \mathrm{m}$ of dendritic length. Considering that the density of dopamine varicosities in the medial PFC rats is 100 times less than in the striatum $\left(1.0 \times 10^{6}\right.$ versus $1.0 \times 10^{8}$ per $\mathrm{mm}^{3}$ of tissue, respectively; Doucet et al., 1986; Descarries et al., 1987), a theoretical synaptic density for rodent cortex would equate to $\sim 0.75$ per $100 \mu \mathrm{m}$ of cortical dendrite. This estimate is in a good agreement with our findings in primate PFC and indicates that TH synaptic appositions may be remarkably highly conserved across structures (cortex and caudate) and species (rat and monkey).

\section{TH innervation of nonpyramidal neurons}

The quantitative data obtained in the present study also allowed us to compute the proportion of all $\mathrm{TH}$ contacts made on pyramidal versus nonpyramidal cells in area $9 \mathrm{~m}$ of the primate PFC. Interneurons comprise only $20-30 \%$ of the neuronal population in the cortical layers (Hendry et al., 1987), and their dendritic tree is, at most, one-half as large as that of pyramidal cells. Therefore, the proportion of TH appositions to spiny versus smooth dendrites taken from our results can be approximated to 91 versus $9 \%$, respectively. Sesack et al. (1995) reported $74 \%$ of TH synapses (and "nonsynaptic" appositions) on spiny dendrites versus $26 \%$ on GABAergic dendrites, based on EM sampling. The small difference between the two studies may be attributable to the fact that Sesack et al. (1995) focused on layers I-III, which could harbor a $>30 \%$ proportion of GABAergic cells [for instance, in layer I GABA cells form $95-100 \%$ of the cell population (Jones, 1993)], whereas our data are exclusively from layer III interneurons. The difference between studies also possibly reflects unpredictable sampling of dopamine and GABA-immunopositive structures because of the poor penetration of antibody in tissue prepared for EM.

\section{Functional implications}

The present findings are relevant to the cortical pathology of the frontal lobe in schizophrenia. Layer II neurons are compromised selectively in cortical regions that receive a strong dopaminergic innervation (Benes et al., 1991; L. Selemon, G. Rajkowska, and P. S. Goldman-Rakic, unpublished observation), and a selective decrease in the density of "extra large" pyramids in layer III also has been reported in schizophrenic brain (Rajkowska et al., 1997). Remarkably, in the present study, the two layer II cells and the one large superficial layer III cell had at least twice the $\mathrm{TH}$ apposition density of any other pyramidal cells. It is tempting to speculate that the density of the dopamine innervation on neurons in layer II and upper layer III may be relevant to these neuropathological findings, because they could provide a direct link between the dopamine innervation of the cortex and cellular pathology in schizophrenia.

\section{REFERENCES}

Benes FM, McSparren J, Bird ED, Vincent SL, SanGiovanni JP (1991) Deficits in small interneurons in prefrontal and anterior cingulate cortex of schizophrenic and schizoaffective patients. Arch Gen Psychiatry 48:996-1001.

Buhl EH, Schwerdtfeger WK, Germroth P (1990) Intracellular injection in fixed brain tissue combined with other neuroanatomical techniques at the light and electron microscopic level. In: Handbook of chemical neuroanatomy analysis of neuronal microcircuits and synaptic interactions, Vol 8 (Björklund A, Hökfelt T, Wouterlood FG, van den Pol, eds), pp 273-304. The Netherlands: Elsevier BV.

Carr DB, Sesack SR (1996) Hippocampal afferents to the rat prefrontal cortex: synaptic targets and relation to dopamine terminals. J Comp Neurol 369:1-15.

Cepeda C, Radisavijevic Z, Peacock W, Levine MS, Buchwald NA (1992) Differential modulation by dopamine of responses evoked by excitatory amino acids in human cortex. Synapse 11:330-341.

Descarries L, Lemay B, Doucet G, Berger B (1987) Regional and laminar density of the dopamine innervation in adult rat cerebral cortex. Neuroscience 21:807-824.

Descarries L, Seguela P, Watkins C (1991) Nonjunctional relationships of monoamine axon terminals in the cerebral cortex of adult rat. In: Volume transmission in the brain (Fuxe K, Agnati LF, eds), pp 53-62. New York: Raven.

Dodt H-U, Zieglgänsberger W (1990) Visualizing unstained neurons in living brain slices by infrared DIC-videomicroscopy. Brain Res 537:333-336.

Dodt H-U, Zieglgänsberger W (1994) Infrared videomicroscopy: a new look at neuronal structure and function. Trends Neurosci 17:453-458.

Doucet G, Descarries L, Garcia S (1986) Quantification of the dopamine innervation in adult rat neostriatum. Neuroscience 19:427-445.

Einstein G (1988) Intracellular injection of Lucifer yellow into cortical neurons in lightly fixed sections and its application to human autopsy material. J Neurosci Methods 26:95-103.

Freund TF, Powell JF, Smith AD (1984) Tyrosine hydroxylaseimmunoreactive boutons in synaptic contact with identified striatonigral neurons, with particular reference to dendritic spines. Neuroscience 13:1189-1215.

Garris PA, Wightman RM (1994) Different kinetics govern dopaminergic transmission in the amygdala, prefrontal cortex, and striatum: an in vivo voltammetric study. J Neurosci 14:442-450.

Garris PA, Ciolkowski EL, Pastore P, Wightman RM (1994) Efflux of dopamine from the synaptic cleft in the nucleus accumbens of the rat brain. J Neurosci 14:6084-6093.

Gaspar P, Berger B, Febvret A, Vigny A, Henry JP (1989) Catecholamine innervation of the human cerebral cortex as revealed by comparative immunohistochemistry of tyrosine hydroxylase and dopaminebeta-hydroxylase. J Comp Neurol 279:249-271.

Geijo-Barrientos E, Pastore C (1995) The effects of dopamine on the subthreshold electrophysiological responses of rat prefrontal cortex neurons in vitro. Eur J Neurosci 7:358-366.

Goldman-Rakic PS (1992) Dopamine-mediated mechanisms of the prefrontal cortex. Semin Neurosci 4:149-159.

Goldman-Rakic PS, Leranth C, Williams SM, Mons N, Geffard M (1989) Dopamine synaptic complex with pyramidal neurons in primate cerebral cortex. Proc Natl Acad Sci USA 86:9015-9019.

Goldman-Rakic PS, Lidow MS, Smiley JF, Williams MS (1992) The anatomy of dopamine in monkey and human prefrontal cortex. J Neural Transm Suppl 36:163-177.

Hendry SHC, Jones EG, Schwark HD, Yan J (1987) Numbers and proportions of GABA-immunoreactive neurons in different areas of monkey cerebral cortex. J Neurosci 7:1503-1519.

Jedema PH, Moghaddam B (1996) Characterization of excitatory amino acid modulation of dopamine release in the prefrontal cortex of conscious rats. J Neurochem 66:1448-1453.

Jones EG (1993) GABAergic neurons and their role in cortical plasticity in primates. Cereb Cortex 3:361-372.

Kawagoe KT, Garris PA, Wiedemann DJ, Wightman RM (1992) Regulation of transient dopamine concentration gradients in the microenvironment surrounding nerve terminals in the rat striatum. Neuroscience 51:55-64. 
Law-Tho D, Hirsch JC, Crepel F (1994) Dopamine modulation of synaptic transmission in rat prefrontal cortex: an in vitro electrophysiological study. Neurosci Res 21:151-160.

Lewis DA, Campbell MJ, Foote SL, Goldstein M, Morrison JH (1987) The distribution of tyrosine hydroxylase-immunoreactive fibers in primate neocortex is widespread but regionally specific. J Neurosci 7:279-290.

Lewis DA, Foote SL, Goldstein M, Morrison JH (1988) The dopaminergic innervation of monkey prefrontal cortex: a tyrosine hydroxylase immunohistochemical study. Brain Res 449:225-243.

MacVicar BA (1984) Infrared video microscopy to visualize neurons in the in vitro brain slice preparation. J Neurosci Methods 12:133-139.

Penit-Soria J, Audinat E, Crepel F (1987) Excitation of rat prefrontal cortical neurons by dopamine: an in vitro electrophysiological study. Brain Res 425:263-274.

Rajkowska G, Selemon LD, Goldman-Rakic PS (1997) Neuronal atrophy in schizophrenic prefrontal cortex contrasts with generative processes in Huntington's disease. Arch Gen Psychiatry, in press.

Sawaguchi T, Goldman-Rakic PS (1994) The role of D1 dopamine receptor in working memory: local injections of dopamine antagonists into the prefrontal cortex of rhesus monkeys performing an oculomotor delayed-response task. J Neurophysiol 71:515-528.

Sesack SR, Snyder CL, Lewis DA (1995) Axon terminals immunola- beled for dopamine or tyrosine hydroxylase synapse on GABAimmunoreactive dendrites in rat and monkey cortex. J Comp Neurol 363:264-280.

Smiley JF, Goldman-Rakic PS (1993) Heterogeneous targets of dopamine synapses in monkey prefrontal cortex demonstrated by serial section electron microscopy: a laminar analysis using the silverenhanced diaminobenzidine sulfide (SEDS) immunolabeling technique. Cereb Cortex 3:223-238.

Smiley JF, Williams SM, Szigeti K, Goldman-Rakic PS (1992) Light and electron microscopic characterization of dopamine-immunoreactive axons in human cerebral cortex. J Comp Neurol 321:325-335.

Stuart GJ, Dodt H-U, Sakmann B (1993) Patch-clamp recordings from the soma and dendrites of neurons in brain slices using infrared video microscopy. Pflügers Arch 423:511-518.

Williams GV, Goldman-Rakic PS (1995) Modulation of memory fields by dopamine D1 receptors in prefrontal cortex. Nature 376:572-575.

Williams SM, Goldman-Rakic PS (1993) Characterization of the dopaminergic innervation of the primate frontal cortex using a dopaminespecific antibody. Cereb Cortex 3:199-222.

Yang CR, Seamans JK (1996) Dopamine D1 receptor actions in layers V-VI rat prefrontal cortex neurons in vitro: modulation of dendriticsomatic signal integration. J Neurosci 16:1922-1935. 